\title{
Improving the Quality of Venous Blood Sampling Procedure (Phlebotomy): Avoiding Tourniquet Use
}

\author{
Francisco Freitas ${ }^{1, \#(0)}$ Mónica Alves ${ }^{2, \#}$ \\ ${ }^{1}$ Microbiology Laboratory, Clinical Pathology Service, Centro \\ Hospitalar Tondela-Viseu, Viseu, Portugal \\ ${ }^{2}$ Central Laboratory, Clinical Pathology Service, Centro Hospitalar \\ Tondela-Viseu, Viseu, Portugal
}

Address for correspondence Francisco Freitas, BSc, MSc, Microbiology Laboratory, Clinical Pathology Service, Centro Hospitalar Tondela-Viseu, Av. Rei Dom Duarte, 3504-509 Viseu, Portugal (e-mail: francyske@gmail.com).

\begin{abstract}
Keywords

- tourniquet application

- phlebotomy

- venous blood sampling

- guidelines

- preanalytical phase

- quality improvement

Background Phlebotomy guidelines discourage tourniquet use whenever possible. We assessed phlebotomists' capability of not using the tourniquet in venous blood sampling, hypothesizing it to be equal to $50 \%$ of the patients attended, and identifying the most frequent venipuncture site.

Materials and Methods We assigned two phlebotomists of the same age (41 years) and experience ( 20 years) to record 10 phlebotomy days, the first with prioritized and the latter with nonprioritized patients. Each acquired daily data for the number of attended patients, age, gender, frequency of nontourniquet usage, and punctured vein. To test our work hypothesis we used the two-tailed single sample $t$-test. Differences between age-group means and nontourniquet use means by each phlebotomist were tested by two-tailed $t$-test for independent means.

Results In 10 phlebotomy days, 683 patients were attended (males 43.2\%). We found no statistically significant difference between age-group means. The combined capability of nontourniquet use was $50.5 \%$, which did not differ from our null hypothesis, but the difference in individual group means was statistically significant, the means being $33 \%$ and $66.9 \%$ (prioritized vs. nonprioritized). The medial cubital vein was the most prone to be punctured (77.7\%).

Conclusion Performing phlebotomies without tourniquet was possible in at least half of the attended patients, though it was more limited in specific group populations.
\end{abstract}

\section{Introduction}

Phlebotomy (venous blood sampling) is probably the most common invasive procedure performed in health-care settings. It is a fundamental basis for surveillance, diagnosis, and treatment of patients and is part of the preanalytical

\footnotetext{
\# Both Francisco Freitas and Mónica Alves are currently biomedical scientists at the Clinical Pathology Service, Centro Hospitalar Tondela-Viseu, Viseu, Portugal.
}

published online September 20, 2021
DOI https://doi.org/ $10.1055 / \mathrm{s}-0041-1735584$ ISSN 0974-2727. phase (before the sample is analyzed in a laboratory). In Portugal, phlebotomy is a part of the daily routine work mainly of nonmedical personnel like nurses and biomedical scientists (BMS) from now on referred as phlebotomists. In an aging world, this procedure is becoming more frequent and sometimes more difficult due to patient's multiple morbidities and/or disabilities.

Several guidelines or recommendations are available to standardize venous blood sampling practice, like the Joint

(c) 2021. The Indian Association of Laboratory Physicians. All rights reserved.

This is an open access article published by Thieme under the terms of the Creative Commons Attribution-NonDerivative-NonCommercial-License, permitting copying and reproduction so long as the original work is given appropriate credit. Contents may not be used for commercial purposes, or adapted, remixed, transformed or built upon. (https://creativecommons.org/ licenses/by-nc-nd/4.0/)

Thieme Medical and Scientific Publishers Pvt. Ltd., A-12, 2nd Floor, Sector 2, Noida-201301 UP, India 
EFLM-COLABIOCLI $^{1}$ or the CLSI GP41-A7 standard, ${ }^{2}$ with both having a detailed step-by-step approach.

Here we point out to the tourniquet application step, which we usually use less than a minute to constrict venous circulation, gaining better vein location and access. Both of the above guidelines discourage its use whenever possible, due to the effects on hemoconcentration, which can lead to spurious results ${ }^{3-6}$ or possible hemolysis due to prolonged venous stasis, ${ }^{7,8}$ thus impacting on patient safety and results turnaround time.

In our laboratory, phlebotomists are sensitive to tourniquet application time and proceed to early release after needle insertion, but only a few are prone to let the tourniquet out of process whenever possible. In light of this, and since there is a gap of data in the literature, the aim of the present study was to assess the phlebotomists capability of not using the tourniquet in real-life blood sampling procedures for diagnostic purposes. We hypothesized that our capability of nontourniquet use equals $50 \%$ of attended patients. We also aim to identify the most frequent venipuncture site without tourniquet use.

\section{Materials and Methods}

\section{Subjects and Materials}

Our laboratory has five boxes for blood sampling procedures, three for nonprioritized and two for prioritized patients. In the first group, we usually attend general outpatients with a first medical consultation or in follow-up for an elective surgery or under oral anticoagulants. In the second group, as per the Portuguese law public hospitals should give priority to outpatients with physical (in wheel chair/litter/other) or mental disabilities, pregnant women, and people accompanied by infants. Our laboratory also prioritizes patients with type I diabetes, with prescribed examinations made in an external laboratory or another following examination in the hospital, and children (6-12 years). Other outpatients are attended only after these.

To standardize the evaluation of tourniquet application and reduce bias between patient groups, we selected and assigned two phlebotomists of the same age (41 years) and experience (20 years), one for each group.

No informed consent or ethical approval was required for this study, as no specific patient information is presented.

\section{Methods}

In a 2-day practical coaching period, phlebotomists were instructed to avoid tourniquet use whenever possible, by asking every patient to make a fist first (without pumping), to assess the presence of prominent veins (prioritizing antecubital), and the possibility of a direct phlebotomy. When blood flow starts, the fist shall then be open. If not possible or confident enough to perform, phlebotomy should be made using the tourniquet. In general, our blood sampling procedure follows the steps of EFLM-COLABIOCLI recommendations. ${ }^{1}$

The study was conducted between April 2 and May 10, 2019, a period in which both phlebotomists recorded
10 phlebotomy days. In a simple record form, each acquired daily data for the number of attended patients, age and gender, the frequency of nontourniquet usage, and the punctured vein.

\section{Statistical Analysis}

To test our work hypothesis, we used relative and combined frequencies of the attended patients without tourniquet use, and tested the difference with the two-tailed single sample $t$-test $(p<0.05)$. The Kolmogorov-Smirnov test was used to assess the normality of distribution of investigated variables (age and nontourniquet use), which were considered to be normally distributed. Differences between age-group means and nontourniquet use means by each phlebotomist were tested by two-tailed $t$-test for independent means $(p<0.05)$. To present data on the punctured vein without tourniquet use, we used absolute count and relative frequencies. Statistical analysis was done using SPSS version 20 software (IBM Corporation, Armonk, New York, United States).

\section{Results}

The main results of the present study are summarized in - Table 1. In 10 phlebotomy days, both phlebotomists attended 683 patients (plus 23 in nonpriority group), with males representing $43.2 \%$ of the total population. We found no statistically difference between age-group means $(p=0.334)$. Considering the nontourniquet use, we verified that the individual means were statistically different ( $p \leq 0.001$ ), with phlebotomist 1 having all of 10 days above $53 \%$, while phlebotomist 2 had only approximately $42 \%$ in 2 days. The combined capability of nontourniquet use was shown to be $50.5 \%$ (345 out of 683 attended patients), which did not differ from our null hypothesis $(p=0.909)$. None of the phlebotomists reported a failed phlebotomy when not using the tourniquet.

As stated in - Table 2, the medial cubital vein was the most prone $(77.7 \%)$ to be punctured when tourniquet was not used, followed by the basilica and cephalic veins, respectively, with both phlebotomists presenting the same pattern.

\section{Discussion}

To our knowledge, this is the first study reporting on the real capability of nontourniquet use when performing a phlebotomy, which we have shown to be $50.5 \%$ of attended patients, confirming our working hypothesis. This value will set the benchmark for future studies on this problematic. Since our laboratory has three boxes for nonprioritized and two for prioritized patients, with appropriate educational interventions we can project a potentially nontourniquet phlebotomy ratio well above $50 \%$.

The medial cubital vein was paramount to our results (-Table 2), because it is the most prominent and easy to puncture, usually does not roll under the skin, and is also a good choice to prevent nerve injury and an hemolyzed sample. ${ }^{1,9-11}$ These results are in clear contradiction with the GP41-A7 standard, which declares that the phlebotomist 
Table 1 Results of NTU in attended groups by each phlebotomist

\begin{tabular}{|c|c|c|c|c|c|c|c|c|c|c|}
\hline \multirow[b]{3}{*}{ Day } & \multicolumn{5}{|c|}{ Phlebotomist 1} & \multicolumn{5}{|c|}{ Phlebotomist 2} \\
\hline & \multicolumn{5}{|c|}{ Nonpriority group } & \multicolumn{5}{|c|}{ Priority group } \\
\hline & TP & Male & Age (y) & NTU, $n$ & NTU, \% & TP & Male & Age (y) & NTU, $n$ & NTU, \% \\
\hline 1 & 35 & $18 / 35$ & $58(18-85)$ & 20 & 57.1 & 27 & $10 / 27$ & $57(11-80)$ & 7 & 26 \\
\hline 2 & 36 & $15 / 36$ & $55(8-93)$ & 20 & 55.5 & 37 & $19 / 37$ & $57(18-91)$ & 7 & 19 \\
\hline 3 & 34 & $14 / 34$ & $61(8-92)$ & 23 & 67.6 & 34 & $16 / 34$ & $55(20-95)$ & 8 & 23.6 \\
\hline 4 & 34 & $14 / 34$ & $54(16-83)$ & 21 & 61.7 & 28 & $10 / 28$ & $60(21-88)$ & 9 & 32.1 \\
\hline 5 & 34 & $16 / 34$ & $54(13-86)$ & 27 & 79.4 & 36 & $17 / 36$ & $58(17-85)$ & 13 & 36.1 \\
\hline 6 & 30 & $13 / 30$ & $59(16-91)$ & 16 & 53.3 & 27 & $16 / 27$ & $61(21-84)$ & 10 & 37 \\
\hline 7 & 28 & $13 / 28$ & $57(34-81)$ & 15 & 53.6 & 24 & $6 / 24$ & $59(29-85)$ & 10 & 41.6 \\
\hline 8 & 43 & $21 / 43$ & $59(27-83)$ & 38 & 88.3 & 34 & $14 / 34$ & $60(12-93)$ & 13 & 38.2 \\
\hline 9 & 39 & $18 / 39$ & $63(33-88)$ & 28 & 71.8 & 37 & $12 / 37$ & $63(21-92)$ & 13 & 35.1 \\
\hline 10 & 40 & $17 / 40$ & $60(20-86)$ & 28 & 70 & 46 & $16 / 46$ & $63(11-96)$ & 19 & 41.3 \\
\hline Total & 353 & $159 / 353$ & $58(8-93)$ & 236 & 66.9 & 330 & $136 / 330$ & $59(11-96)$ & 109 & 33 \\
\hline
\end{tabular}

Abbreviations: NTU, nontourniquet use; TP, total patients attended.

Table 2 Punctured veins by each phlebotomist without tourniquet use

\begin{tabular}{|l|l|l|l|l|}
\hline & \multicolumn{3}{|l|}{ Veins } & \\
\hline & $\begin{array}{l}\text { Medial } \\
(n, \%)\end{array}$ & $\begin{array}{l}\text { Basilic } \\
(n, \%)\end{array}$ & $\begin{array}{l}\text { Cephalic } \\
(n, \%)\end{array}$ & Total \\
\hline Phlebotomist 1 & $185(78.4)$ & $32(13.6)$ & $19(8)$ & 236 \\
\hline Phlebotomist 2 & $83(76.2)$ & $14(12.8)$ & $12(11)$ & 109 \\
\hline Total $(n, \%)$ & $268(777)$ & $46(13.3)$ & $31(9)$ & 345 \\
\hline
\end{tabular}

may not be able to prioritize the antecubital veins without tourniquet application. ${ }^{2}$

Currently, there is no study with which we can compare our results, but as stated by Lima-Oliveira et al, approximately $78 \%$ of phlebotomies in outpatients are performed in the medial cubital vein when using a tourniquet, ${ }^{12}$ and we have achieved similar results without its use. Adding to this, neither phlebotomist had a failed phlebotomy, which demonstrates good selection and confidence to perform.

We have also confirmed that performing phlebotomy in prioritized patients limits our capability of drawing blood without tourniquet use, despite no difference found between age-group means. This clearly shows that we stand before two different group-populations.

Other interventions to avoid tourniquet application like transillumination devices have been tested, and proved to be useful in eliminating venous stasis and improving the quality of phlebotomy procedures, especially critical in patients with difficult or small veins, such as children and old people. ${ }^{5,13}$ Despite the advantages, this technology has not paved its way into the health-care setting, which is also our case scenario.

Three limitations need to be acknowledged regarding the present study. The first is that each phlebotomist has their own technique and confidence to perform, and we cannot measure their effect on the results. The second limitation is the number of phlebotomy days. As stated in - Table 1, phlebotomist 2 had better and maintained values of nontourniquet use since day 5 . The third one is that we have not tested the reverse group-phlebotomist pair to verify that the results were still concordant.

\section{Conclusion}

This is the first study reporting on the real capability of nontourniquet use when performing a phlebotomy, which we have shown to be possible and desirable in at least half of the attended patients, though it is more limited in specific group populations. We also demonstrate that phlebotomists were able to prioritize the antecubital veins without tourniquet application, with the medial cubital vein the most prone to be punctured (78\% of attempts). Our results clearly provide some room for continuous quality improvement in the laboratory pre-analytical phase, one of the primary sources of errors in the total testing process. We also set the benchmark for future studies on this problematic that impacts phlebotomy practice, especially in outpatient settings.

\section{Highlights}

- Phlebotomy (venous blood sampling) is probably the most common invasive procedure performed in health-care setting; it is a fundamental basis for surveillance, diagnosis and treatment of patients; and it is part of the laboratory preanalytical phase.

- Since there is a gap of data in the literature, the aim of the present study was to assess the phlebotomists' capability of not using the tourniquet in real-life blood collection 
procedures for diagnostic purposes, which the guidelines recommend to be whenever possible, but professionals rarely have that in account.

- In the study period, phlebotomists safely collected blood from at least half (50.5\%) of the attended patients without tourniquet use, and were also able to prioritize the antecubital veins (medial cubital vein the most prone to be punctured-78\% of attempts).

- Our results will set the benchmark for future studies on this problematic, and may have an impact in phlebotomy practice, especially in outpatient settings.

\section{Authors' Contributions}

FF and MA made a substantial contribution to the concept or design of the work, acquisition, analysis, and interpretation of data. They also drafted the article or revised it critically for important intellectual content. They approved the version to be published. FF and MA participated sufficiently in the work to take public responsibility for appropriate portions of the content. The manuscript is not under review of any other journal, and that it has not been published complete or partially in any other journal, though a previous version has already a DOI in medRxiv (doi.org/10.1101/2020.04.05.20039560).

\section{Conflicts of Interest}

None to declare.

\section{References}

1 Simundic AM, Bölenius K, Cadamuro J, et al; Working Group for Preanalytical Phase (WG-PRE), of the European Federation of Clinical Chemistry and Laboratory Medicine (EFLM) and Latin American Working Group for Preanalytical Phase (WG-PRE-LATAM) of the Latin America Confederation of Clinical Biochemistry (COLABIOCLI) Joint EFLM-COLABIOCLI recommendation for venous blood sampling. Clin Chem Lab Med 2018;56(12):2015-2038
2 Clinical and Laboratory Standards Institute. GP41: Procedures for the Collection of Diagnostic Blood Specimens by Venipuncture; Approved Standard-Seventh Edition. CLSI document GP41-A7. Wayne, PA: Clinical and Laboratory Standards Institute; 2017

3 Lima-Oliveira G, Lippi G, Salvagno GL, et al. Venous stasis and whole blood platelet aggregometry: a question of data reliability and patient safety. Blood Coagul Fibrinolysis 2015;26(06):665-668

4 Lima-Oliveira G, Lippi G, Salvagno GL, Montagnana M, Picheth G, Guidi GC. The effective reduction of tourniquet application time after minor modification of the CLSI H03-A6 blood collection procedure. Biochem Med (Zagreb) 2013;23(03):308-315

5 Lima-Oliveira G, Lippi G, Salvagno GL, et al. New ways to deal with known preanalytical issues: use of transilluminator instead of tourniquet for easing vein access and eliminating stasis on clinical biochemistry. Biochem Med (Zagreb) 2011;21(02):152-159

6 Lippi G, Salvagno GL, Montagnana M, Brocco G, Guidi GC. Influence of short-term venous stasis on clinical chemistry testing. Clin Chem Lab Med 2005;43(08):869-875

7 Phelan MP, Reineks EZ, Schold JD, Hustey FM, Chamberlin J, Procop GW. Preanalytic factors associated with hemolysis in emergency department blood samples. Arch Pathol Lab Med 2018;142(02):229-235

8 McCaughey EJ, Vecellio E, Lake R, et al. Key factors influencing the incidence of hemolysis: a critical appraisal of current evidence. Crit Rev Clin Lab Sci 2017;54(01):59-72

9 Ohnishi H. How to prevent phlebotomy-related nerve injury. Rinsho Byori 2007;55(03):251-256

10 Mukai K, Nakajima Y, Nakano T, et al. Safety of venipuncture sites at the cubital fossa as assessed by ultrasonography. J Patient Saf 2020;16(01):98-105

11 Lippi G, Avanzini P, Aloe R, Cervellin G. Blood collection from intravenous lines: is one drawing site better than others? Lab Med 2014;45(02):172-175

12 Lima-Oliveira G, Lippi G, Salvagno GL, Picheth G, Guidi GC. Laboratory diagnostics and quality of blood collection. J Med Biochem 2015;34(03):288-294

13 Lima-Oliveira G, Lippi G, Salvagno GL, et al. Transillumination: a new tool to eliminate the impact of venous stasis during the procedure for the collection of diagnostic blood specimens for routine haematological testing. Int J Lab Hematol 2011;33(05): 457-462 